\title{
Measurement of III-V Compound Semiconductor Characteristics using the Contactless Electroreflectance Method
}

\author{
Jae-In Yu*, Soon-Don Choi ${ }^{\dagger}$ and Ho-Gyeong Chang**
}

\begin{abstract}
The electromodulation methods of photoreflectanceand the related technique of contactless electroreflectance(CER) are valuable tools in the evaluation of important device parameters for structures such as heterojunction bipolar transistors, pseudomorphic high electron mobility transistors, and quantum dots(QDs). CER is a very general principle of experimental physics. Instead of measuring the optical reflectance of the material, the derivative with respect to a modulating electric field is evaluated. This procedure generates sharp, differential-like spectra in the region of interband (intersubband) transitions. We conduct electric-optical studies of both GaAs layers and InAs selfassembled QDs grown by molecular beam epitaxy. Strong GaAsbandgap energy is measured in both structures. In the case of lnAs monolayers in GaAs matrices, the strong GaAsbandgap energy is caused by the lateral quantum confinement.
\end{abstract}

Keywords: InAs, QD, CER, GaAs, Semiconductor

\section{Introduction}

Semiconductor quantum dots (QDs) have attracted considerable interest recently due to their potential in technological applications. Although Stranski-Krastanow growth is regarded as a promising road toward zerodimensional quantum structures, its random nucleation in a single layer results in a broad distribution of QD size and position. QD arrays of the same size and shape are required for practical applications; thus, the identification of growth mechanisms that lead to a narrowing of the size distribution is of great importance [1, 2]. Modulation reflectance spectroscopy is a powerful tool that investigatesthe optical properties of III-V compound semiconductors. Since the modulation reflectivity is an absorption-type technique, the acquired information reflects the optical transition behavior, which is more directly related to the theoretical absorption model. The optical properties of InAs/GaAs QDs are studied by contactless electroreflectance (CER) and scanning electron microscopy.

\section{Theory}

Modulation spectroscopy is an important technique for the study and characterization of semiconductor thin films and heterostructures. Techniques such as CER and

$\dagger \quad$ Corresponding Author: School of Materials Science and Engineering, Yeungnam University, Korea.

* School of Materials Science and Engineering, Yeungnam University, Korea. (yji5945@yumail.ac.kr)

** Department of Oriental Biomedical Engineering, DaeguHanny University, Korea. (changhg@dhu.ac.kr)

Received: September 30, 2010; Accepted: January 28, 2011 photoreflectance are very useful in probing the bandstructure of the semiconductor becausethey measure the differential reflectance change and are sensitive to surface or interface electric fields. They are particularly useful techniquesfor probingthe band structure of the semiconductors when conventional techniques such as photoluminescence cannot be used because of its bad sample quality. CER requires no special sample mounting and can be performed in a variety of transparent ambiance because it is contactless. In CER measurement, the sample is placed between two capacitor plates. The size of the spacer is such that there is a very thin layer of air between the front surface of the sample and the conducting part of the first electrode. There is nothing in direct contact with the front surface of the sample. The CER spectra as a function of photon energy can be fitted using a familiar Aspnes derivative function in the low electric field limit [3-5]

$$
\frac{\Delta R}{R}=\operatorname{Re} \sum_{j=1}^{p} C_{j} e^{i \theta_{j}}\left(E-E_{g i}=i \Gamma_{j}\right)^{-n}
$$

In the above equation, $R$ is the reflectance, $\Delta \mathrm{R}$ is the induced change in the reflectance by modulation light, $E$ is the photon energy, and $p$ is the total number of spectral structures to be fitted. $E_{g j}, \Gamma_{j}, C_{j}$, and $\theta_{j}$ are the transition energy, broadening parameter, amplitude, and phase of the $j$-th feature corresponding to a critical point, respectively.

\section{Experiment}

The InAs/GaAs QDs structure was grown on undopedGaAs substrates by molecular beam epitaxysystem. After the growth 
of a 500nm-thick GaAs buffer layer at the substrate temperature $\left(\mathrm{T}_{\mathrm{g}}\right)$ of $540^{\circ} \mathrm{C}, \mathrm{InAs} / \mathrm{GaAs}$ QDs structures were grown at $\mathrm{T}_{\mathrm{g}}$ of $460^{\circ} \mathrm{C}$.

The growth sequence for the InAs/GaAs QDs structure is presented in Table 1.

Table 1. The growth structure of InAs/GaAs QDs.

\begin{tabular}{c|c}
\hline GaAs $90 \mathrm{~nm}$ & $0.5 \mathrm{ML} / \mathrm{s}$ \\
\hline GaAs $10 \mathrm{~nm}$ & $0.5 \mathrm{ML} / \mathrm{s}$ \\
\hline As supply & $1 \times 10^{-4} \mathrm{Torr}$ \\
\hline In $4.5 \mathrm{ML}$ & $0.142 \mathrm{ML} / \mathrm{s}$ \\
\hline Ga $1 \mathrm{ML}$ & $0.1 \mathrm{ML} / \mathrm{s}$ \\
\hline GaAs buffer $500 \mathrm{~nm}$ & $0.8 \mathrm{ML} / \mathrm{s}$ \\
\hline UndopedGaAs sub & \\
\hline
\end{tabular}

\section{Result and Discussion}

We begin by analyzing the morphology of the WL formed on deposition of 4.5 ML of InAs on the $\mathrm{GaAs}(001)$ surface. Fig. 1 shows the transitions of A1 $(1.34 \mathrm{eV})$ and A2 $(1.37 \mathrm{eV})$ in CER spectra of the InAs/GaAs QD sample, which are ascribed to the heavy-hole-type and the lighthole-type transitions, respectively.

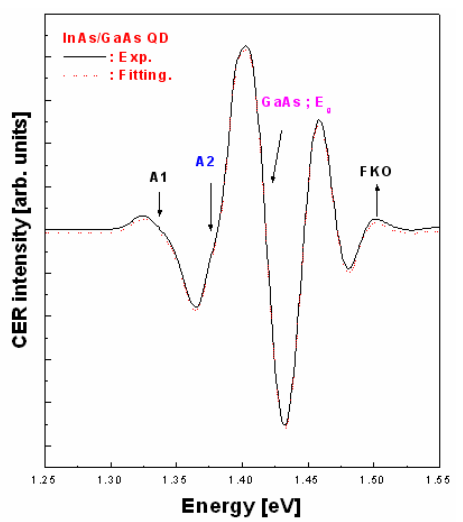

(a)

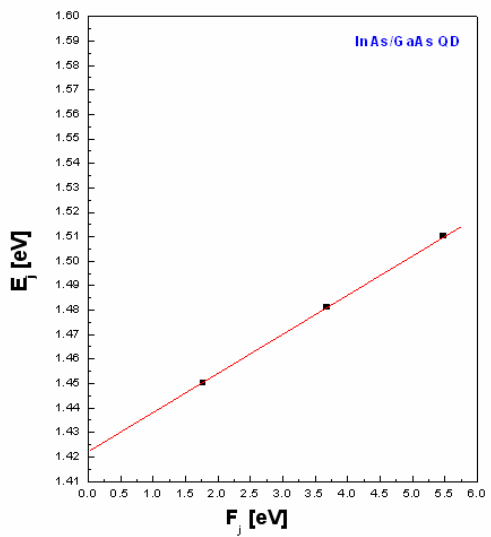

(b)

Fig. 1. The illustrated CER spectrum in InAs/GaAs QDs.
We have

$$
\left[-\frac{1}{r^{2}}\left(r \frac{\partial}{\partial r} r \frac{\partial}{\partial r}+\frac{\partial^{2}}{\partial \theta^{2}}\right)-\frac{\partial^{2}}{\partial z^{2}}+V_{e}(r, z)\right] \Psi(r, \theta, z)=E \Psi(r, \theta, z)
$$

Wave function can be given by two sub-functions, slowly varying function $g_{r}(z)$ of $r$ and $f_{m}(r)$, which fit a set of equations for each angular momentum channel $m$ :

$$
\begin{gathered}
{\left[-\frac{\partial^{2}}{\partial z^{2}}+V_{e}(r, z)\right] g_{r}=E_{o}(R) g_{r}(z),} \\
{\left[-\frac{1}{r^{2}}\left(r \frac{\partial}{\partial r} r \frac{\partial}{\partial r}+m^{2}\right)-E_{o}(r)\right] f_{m}(r)=E f_{m}(r)}
\end{gathered}
$$

Then, the potential distribution of the conductive bandedge within the eight-band KP theory model derived by Califano et al. $[6,7]$ can be expressed as

$$
V_{e}=E_{v, a v}+\frac{\Delta_{o}}{3}+E_{g, o}+a_{e} e_{h h}
$$

In addition, that of the heavy hole is

$$
V_{h h}=E_{v, a v}+\frac{\Delta_{o}}{3}+a_{e} e_{h h}-b e_{t}
$$

where $E_{v, a v}$ is the average valence-band energy; $\Delta_{\mathrm{o}}$ is the spin-orbit splitting; $a_{e}$ and $b$ are the deformation potentials; and $e_{h h}$ and $e_{t}$ are the hydrostatic and uniaxial strain, respectively. This result is shown in Table 2.

Table 2. Fitting experimental values and calculation results with effective mass approximation.

\begin{tabular}{c|c|c}
\hline \multicolumn{3}{|c}{ InAs/GaAs QDs } \\
\hline \multirow{2}{*}{ Peak } & $\begin{array}{c}\text { CER } \\
\text { [Transition } \\
\text { E(eV);300K] }\end{array}$ & $\begin{array}{c}\text { Calculation } \\
{[\text { Transition }} \\
(\mathrm{eV}) ; 300 \mathrm{~K}]\end{array}$ \\
\hline A1 & 1.344 & 1.347 \\
\hline A2 & 1.375 & 1.382 \\
\hline
\end{tabular}

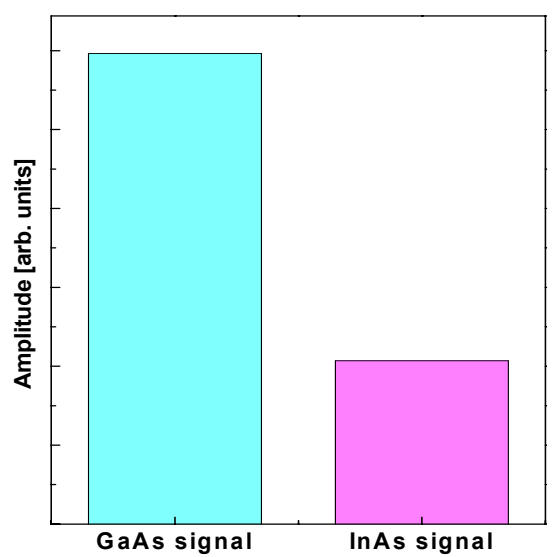

Fig. 2. The graph of amplitude in GaAs and InAs signals 
The strong GaAsbandgap energy was measured. In the case of the $\ln A s$ monolayer in GaAs matrices, the strong GaAsbandgap energy is caused by the lateral quantum confinement. For the InAs QD sample, additional spectral features appeared as shown in Fig. 3. The critical point (CP) in the CER spectrum at $1.405 \mathrm{eV}$ exhibits an emission of red-shift of $\sim 11 \mathrm{meV}$ compared with that of the annealing InAs QD sample. The annealing sample InAs QD disappearedat the A1 and A2 peaks. This result was caused by surface damage [8-12].

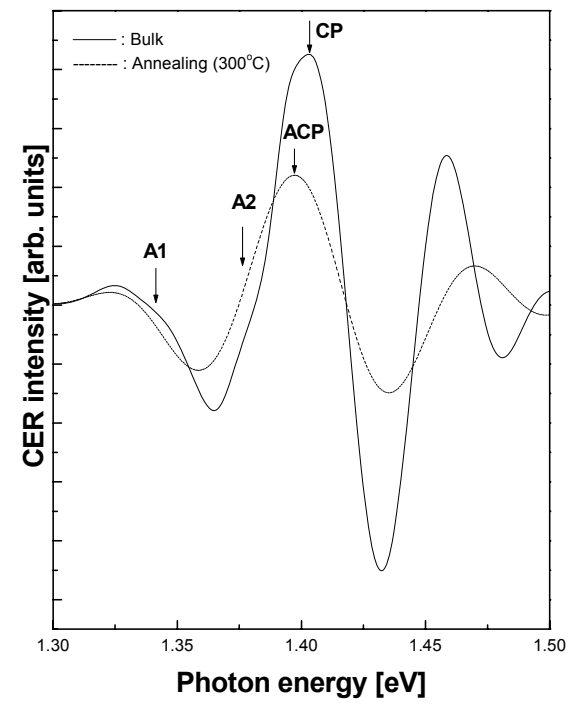

Fig. 3. The CER spectra in InAs QD and annealing InAs QD sample.

Fig. 4 shows anoptical photograph of the annealing InAs QD sample $\left(100-400{ }^{\circ} \mathrm{C}\right)$.

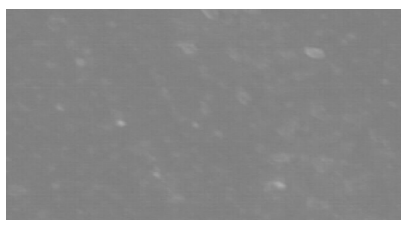

$100{ }^{\circ} \mathrm{C}$

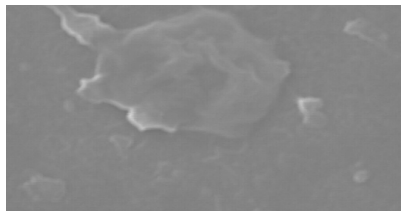

$300^{\circ} \mathrm{C} /$ surface damage

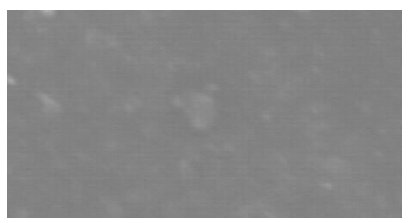

$200{ }^{\circ} \mathrm{C}$

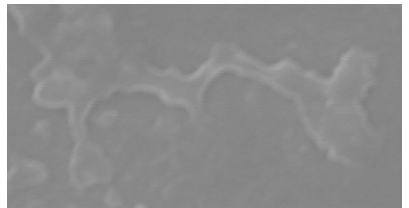

$400{ }^{\circ} \mathrm{C}$
Fig. 4. The photograph of the annealing InAs QD sample.

\section{Conclusion}

We investigated the CER spectra in the InAs QD sample. The CER result and the strong GaAsbandgap energy were measured. In the case of the $\ln A s$ monolayer in GaAs matrices, the strong GaAsbandgap energy was caused by the lateral quantum confinement. The $\mathrm{CP}$ in the CER spectrum at 1.405 eVexhibitedan emission of red-shift of $\sim 11 \mathrm{meV}$ compared with that of the annealing InAs QD sample. This result was caused by surface damage.

\section{Acknowledgments}

This research was supported by the Yeungnam University research grants in 2009.

\section{References}

[1] G.P. Kothiyal, S. Hong, N. Debbar, P.K. Bhattacharya, J. Singh, Appl. Phys. Lett. 51 (1987), p1091.

[2] D.J. Aren, K. Deneffe, C. Van Hoof, J. De Broeck, G. Borfhs, In band structure engineering of semiconductor microstructure, in: R.A. Abram, M. Jacos (Eds.), NATO ARW Series, vol. 189, Plenum, New York, 1988.

[3] S.H. Pan, H. Shen, Z. Hang, F.H. Pollak, A.P. Roth, D. Morris, Phys. Rev. B 38 (1988), p 3375.

[4] B.G. Orr, D. Kessler. C.W. Snyder, L. Sander, Europhys. Len. 19 (1992), p33.

[5] D. Leonard, K. Pond, P.M. Petroft, Phys. Rev. B 50 (1994), p11687.

[6] S.P. DenBaars, C.M. Reaves, V. Bressler-Hill, S. Varma, W.H. Weinberg, P.M. Petroff, J. Cryst. Growth 145(1994), p 721.

[7] R.L. Harper Jr., R.N. Bicknell, D.K. Blanks, N.C. Giles, J.F. Schetzina, Y.R. Lee, A.K. Ramdas, J. Appl. Phys. 65 (1989), p624.

[8] A. Poliment, A. Patane, M. Henini, L. Eaves, P.C. Main, Phys. Rev. B 59 (1999), p5064.

[9] A.E. Zhukov, A.R. Kovsh, N.A. Maleev, S.S. Mikhrin, V.M. Ustinov, M.V. Maximov, Z.I. Alferov, N.N. Ledentsov, D. Bimberg,Appl. Phys. Lett. 75 (1999), p1926.

[10] K.S. Stevens, M. Kinninburgh and R. Beresford, Appl. Phys. Lett. 66 (1995), p3518.

[11] J.M. Gerard, J.B. Genin, J. Lefebvre, J.M. Moison, N. Lebouche and F. Barthe, J. Cryst. Growth 150 (1995), p351.

[12] M. Zachau, P. Helgesen, F. Koch, D. Grutzmacher, R. Meyer and P. Balk, Sernicond. Sci. Technol. 3 (1988), p 1029. 


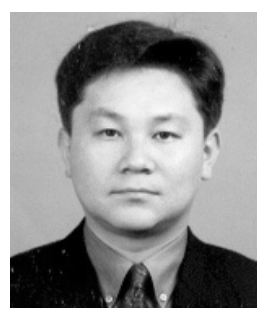

Jae-In Yu received his M.S degree in Physics of Semiconductor from Yeungnam University. He completed Ph.D. in Yeungnam University. He also workedas a researcherin Korea Institute of Machine \& Materials and as chief of YU Electronics (2005-2006), TYE Research (2006-2009), and SEMIYU Research (2009-2011). He currently serves as thehead of Wiscohitec Research. He is also a professor inthe Department of Oriental Biomedical Engineering, Daegu Hanny University, Korea.

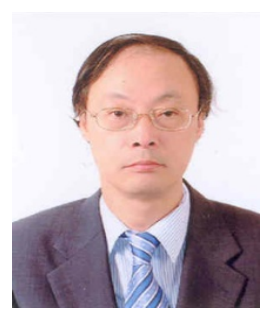

Soon-Don Choi obtained his B.S. and M.S. degrees from the Department of Materials Science and Engineering, Seoul University. He receivedhis Ph.D. from theDepartment of Materials Science and Engineering, Universityof Michigan, USA. He is a Professor atthe Department of Materials Science and Engineering, Yeungnam University, Korea.

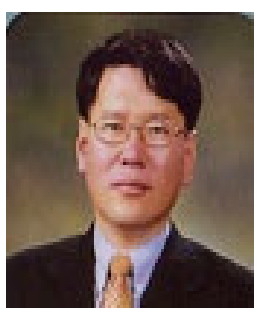

Ho-Gyeong Chang received his B.S., M.S., and Ph.D.degrees from the Department of Physics, Yeungnam University. He is a Professor atthe Department of Oriental Biomedical Engineering, Daegu Hanny University, Korea. 